\title{
Minimizing Total Earliness and Tardiness for Common Due Date Single-Machine Scheduling with an Unavailability Interval
}

\author{
Chinyao Low, ${ }^{1}$ Rong-Kwei $\mathrm{Li}^{2}{ }^{2}$ and Guan-He $\mathrm{Wu}^{2}$ \\ ${ }^{1}$ Department of Industrial Engineering and Management, National Yunlin University of Science and Technology, \\ 123 University Road, Douliou, Yunlin 64002, Taiwan \\ ${ }^{2}$ Department of Industrial Engineering and Management, National Chiao Tung University, 1001 University Road, \\ Hsinchu 30010, Taiwan \\ Correspondence should be addressed to Chinyao Low; lowcy@yuntech.edu.tw
}

Received 23 March 2016; Accepted 6 June 2016

Academic Editor: Miguel A. Salido

Copyright (C) 2016 Chinyao Low et al. This is an open access article distributed under the Creative Commons Attribution License, which permits unrestricted use, distribution, and reproduction in any medium, provided the original work is properly cited.

This paper addresses the problem of scheduling $n$ independent jobs on a single machine with a fixed unavailability interval, where the aim is to minimize the total earliness and tardiness (TET) about a common due date. Two exact methods are proposed for solving the problem: mixed integer linear programming formulations and a dynamic programming based method. These methods are coded and tested intensively on a large data set and the results are analytically compared. Computational experiments show that the dynamic programming method is efficient in obtaining the optimal solutions and no problems due to memory requirement.

\section{Introduction}

This paper considers the minimization of total earliness and tardiness (TET) for common due date single-machine scheduling with an unavailability interval. The TET performance measure is consistent with the just-in-time (JIT) manufacturing philosophy in which an ideal schedule consists of all jobs finish exactly on the due date.

These days, the emphasis on the JIT approach has led to a growing interest in earliness-tardiness scheduling models. In a JIT scheduling environment, job earliness may cause bounded capital and inventory holding costs, whereas job tardiness may disrupt a customer's operations. Therefore, both earliness and tardiness should be taken into account in order to determine the optimal machine scheduling policy.

The common due date scenarios are relevant in many realistic situations; for example, several items constitute a customer order, or they are delivered to an assembly line where the components are all required to be ready at the same time. Numerous studies have been published on the earlinesstardiness scheduling problems with common due date, such as those discussed in the surveys of Baker and Scudder [1], Biskup and Feldmann [2], Gordon et al. [3], and Lauff and
Werner [4]. In what follows, some references related to the common due date TET minimization are recalled.

Kanet [5] proposed a polynomially bounded matching algorithm for the single-machine problem that the due date was assumed to be greater than the total processing time of the jobs. Bagchi et al. [6] extended Kanet's result and provided an implicit enumeration procedure to find all the optimal schedules. Sundararaghavan and Ahmed [7] developed a polynomial-time algorithm to determine the optimal jobmachine assignment for identical parallel machines. Emmons [8] investigated the uniform parallel-machine scheduling problem when the makespan and machine occupancy were considered as the secondary objective. Baker and Scudder [1] specified the minimum value of a common due date that gives rise to the unrestricted version of the TET problem. Dynamic programming algorithms and branch-and-bound methods for single-machine scheduling with a restricted due date were presented in [6,9-13]. Sarper [14] modelled the two-machine flow shop scheduling problem as a mixed integer linear programming formulation and suggested three constructive heuristics. Sakuraba et al. [15] introduced a job timing algorithm for generating the optimal schedule when the sequence of jobs processed in the two-machine flow shop 
was given. Sung and Min [16] addressed the problem of scheduling two-machine flow shop with at least one batch processor incorporated. Some optimality properties were characterized for three different system configurations to derive their corresponding solution methods. Yeung et al. [17] proposed a branch-and-bound method and a heuristic to solve the two-machine flow shop scheduling problem with TET criterion in relation to a given common due window. Lauff and Werner [18] investigated the computational complexity of multistage scheduling problems with intermediate storage costs.

It can be observed that the majority of previous studies assumed that the machines are available at all times. However, in a real production system, machines may not be always available because of unforeseen breakdowns, tool changes, or preventive maintenance requirements for improving the production efficiency. It is also possible that machines have been committed to deal with the promised orders and hence become unavailable for processing in certain periods of the current planning horizon. Under such circumstances, the optimal production strategies can be quite different from those attained by classical models.

Due to the practical experience in production systems, there has been a great deal of efforts concentrated on scheduling problems with limited machine availability (see Lee et al. [19]; Schmidt [20]; Ma et al. [21]). More recently, Low et al. [22] presented heuristic approaches to minimize the makespan on a single machine that was periodically unavailable. Mosheiov and Sidney [23] developed polynomial-time algorithms for scheduling a deteriorating maintenance activity on a single machine with different objective functions. Zhao and Tang [24] analyzed the single-machine scheduling problem with simultaneous considerations of maintenance activities and job-dependent aging effect while minimizing the makespan.

Ángel-Bello et al. [25] proposed a mixed integer programming formulation and an efficient heuristic approach for the single-machine makespan problem with availability constraints and sequence-dependent setup costs. Huo and Zhao [26] presented polynomial-time algorithms to optimize both makespan and total completion time under a twoparallel-machine environment with availability constraints. Yang et al. [27] provided a dynamic programming algorithm and a branch-and-bound method for minimizing the total completion time on a single machine in which job processing and maintenance activity had to be scheduled simultaneously. Lee and Kim [28] studied the single-machine scheduling problem with periodic maintenance and adopted a twophase heuristic to minimize the number of tardy jobs. Yin et al. [29] examined the problem of scheduling jobs and common due date assignment on a single machine with a rate-modifying activity. They proposed some optimality conditions and developed polynomial-time algorithms for special cases.

Dong [30] presented a column generation based branchand-bound method to schedule identical parallel machines in which the job sequence and the timing of shutdown operation were jointly optimized. Hsu et al. [31] considered the scheduling problem of minimizing the total completion time and the total machine load on the unrelated parallel machines with three basic types of aging effect models and deteriorating maintenance activities. They showed that all the addressed models are polynomial-time solvable. Shen et al. [32] proposed polynomial-time algorithms to schedule identical parallel machines with nonsimultaneous machine available time. Vahedi-Nouri et al. [33] developed a new binary integer programming formulation and a branch-and-bound method for minimizing the total completion time in single-machine scheduling with learning effect and multiple availability constraints. $\mathrm{Xu}$ and Yang [34] considered the two-parallel-machine scheduling problem with a periodic availability constraint. They presented a mathematical programming model to minimize the makespan.

Hashemian et al. [35] addressed the makespan minimization for parallel-machine scheduling with multiple planned unavailability intervals. A mixed integer linear programming model and an implicit enumeration algorithm were designed to tackle the problem. Kaplanoğlu [36] adopted a multiagent based approach for scheduling single machine with sequence-dependent setup times and machine maintenance, where both of the regular and irregular maintenance activities were considered. Rustogi and Strusevich [37] studied the single-machine scheduling problem incorporating positional and time-dependent effects, in which the machine was subject to rate-modifying activities that split the jobs into groups. The aims were to minimize the makespan and the total completion time. Yin et al. [38] explored the single-machine batch scheduling problem with an unavailability interval. A dynamic programming algorithm was proposed for minimizing the sum of total flow time and batch delivery cost. Yin et al. [39] investigated the problem of scheduling jobs with assignable due dates on a single machine, in which the job processing times were subject to positional deterioration and the execution of preventive maintenance. They analyzed the structural properties of the problems under consideration and presented polynomial-time algorithms for deriving the optimal solution. Rustogi and Strusevich [40] considered the single-machine scheduling problem with linear timedependent deterioration effects and maintenance activities. A range of polynomial-time algorithms were designed to minimize the makespan.

Most of the aforementioned scheduling models focused on regular performance measures such as makespan, total completion time, and number of tardy jobs. With current emphasis on the JIT production strategy, these classical measures may no longer be applicable. So far, only Low et al. [41] addressed the problem of minimizing common due date TET in the presence of availability constraints. They developed an ant colony optimization algorithm for the single-machine model in which one maintenance task had to be performed and analyzed some special cases that are polynomial-time solvable. Nevertheless, such heuristic approaches offer no guarantees to the optimality of the obtained solutions and do not define how close the obtained solutions are to the optimal ones. Furthermore, it should be noted that the matching algorithm suggested by Low et al. [41] only can be used to deal with the cases when the due 
date falls within the unavailability interval and certain conditions are satisfied. In practical scheduling environments, the production schedulers always expect to come up with an optimal schedule in reasonable time. It might be undesirable to use heuristic approaches to tackle problems while efficient exact algorithms are available. Thus, more powerful optimization techniques must be brought to bear on the problem.

This paper contributes two exact methods: mixed integer linear programming formulations and a dynamic programming method. The mixed integer linear programming formulations are characterized by the type of binary variable that captures the scheduling decision, and the dynamic programming method is derived based on the optimality properties. Experimental results show that the proposed methods are able to give satisfactory solutions. Moreover, it is computationally demonstrated that the dynamic programming method is efficient in obtaining the optimal schedules for large-scale problems.

In the next section, a formal statement of the problem is established and some optimality properties are given. Section 3 provides four mixed integer linear programming formulations based on different definitions of decision variables, while Section 4 describes the dynamic programming algorithms. In Section 5, computational results are reported to evaluate the proposed methods. Finally the conclusions are drawn in Section 6.

\section{Problem Presentation and Optimality Properties}

2.1. Description of the Problem. A set of $n$ independent jobs has to be processed on a single machine which is unavailable in a given time interval $\left[t_{s}, t_{e}\right]$. Each job $j(j=$ $1,2, \ldots, n)$ becomes available for processing at time zero, has a processing time $p_{j}$, and should ideally be completed at a common due date $d$. The machine can handle at most one job at a time and preemption of jobs is prohibited. Without loss of generality, it is assumed that all data are integral. A schedule defines for each job $j$ a completion time $C_{j}$. Let $E_{j}=\max \left\{0, d-C_{j}\right\}$ and $T_{j}=\max \left\{0, C_{j}-d\right\}$ represent the earliness and tardiness, respectively, of job $j$. The objective is to determine a feasible schedule so that the TET about the common due date $\sum_{j=1}^{n}\left|C_{j}-d\right|=\sum_{j=1}^{n}\left(E_{j}+T_{j}\right)$ are minimized. Extending the standard three-field notation in Graham et al. [42], this problem can be referred to as $1, h_{1}$ | $d_{j}=d \mid \sum_{j=1}^{n}\left(E_{j}+T_{j}\right)$, where $h_{1}$ indicates that there is only one unavailability interval on the machine.

2.2. Problem Analysis. In absence of machine availability constraints, the problem under consideration is reduced to the problem $1\left|d_{j}=d\right| \sum_{j=1}^{n}\left(E_{j}+T_{j}\right)$, which has been proven to be NP-hard by Hall et al. [9] based on the evenodd partition problem, and hence the addressed problem is also NP-hard. To begin with, some important optimality properties are established. They are essential for the design of the dynamic programming.
Since the machine cannot process any jobs in an unavailability interval $\left[t_{s}, t_{e}\right]$, there are two available time windows $\delta_{i}=\left[D_{i}, R_{i}\right]$, where

$$
\begin{aligned}
& i=1: D_{i}=0 \text { and } R_{i}=t_{s}, \\
& i=2: D_{i}=t_{e} \text { and } R_{i}=u b C^{*},
\end{aligned}
$$

where $u b C^{*}$ is an upper bound on the makespan. Let $\sigma$ be the index of the job for which $C_{\sigma}-p_{\sigma}<d \leq C_{\sigma}$. Define $J_{E}=\left\{1 \leq j \leq n \mid C_{j} \leq d\right\}$ as the set of jobs that finish on or before the due date, and define $J_{T}=\left\{1 \leq j \leq n \mid C_{j}-p_{j} \geq d\right\}$ as the set of jobs that start on or after the due date. In addition, we denote by $J_{i}=\left\{1 \leq j \leq n \mid C_{j} \leq R_{i}, C_{j}-p_{j} \geq D_{i}\right\}$ the set of jobs that are processed in available time windows $\delta_{i}$, such that $\sum_{j \in J_{i}} p_{j} \leq R_{i}-D_{i}, J_{1} \cup J_{2}=\{1,2, \ldots, n\}$, and $J_{1} \cap J_{2}=\emptyset$. Properties 1 and 4 generalize the results given by Cheng and Kahlbacher [43] and Bagchi et al. [6], respectively. Properties 2 and 3 are straightforward to prove by contradiction. Property 5 is an extension of the weakly Vshaped schedule optimality shown in Hall et al. [9]. It is easily verified that such a result can be generalized to the case with an unavailability interval.

Property 1. In an optimal schedule there are no idle times within the processing of consecutive jobs in each available time window.

Property 2. The earliest job in an optimal schedule must start at or before time $t=\max \left\{d, t_{e}\right\}$.

Property 3. In an optimal schedule, there is no idle interval between the last job in $\delta_{1}$ and time $t_{s}$ when $t_{s} \leq d$, and there is no idle interval between the first job in $\delta_{2}$ and time $t_{e}$ when $t_{e} \geq d$.

Property 4. In an optimal schedule, the jobs in set $J_{E} \cap J_{i}$ are sorted according to nonincreasing order of processing times (LPT), and the jobs in set $J_{T} \cap J_{i}$ are sorted according to nondecreasing order of processing times (SPT), for all $i=$ 1,2 .

Property 5. An optimal schedule must satisfy $p_{\sigma} \leq$ $\max \left\{\min _{j \in J_{E} \cap J_{2}}\left\{p_{j}\right\}, \min _{j \in J_{T}}\left\{p_{j}\right\}\right\}$ if $t_{e}<d$ or $p_{\sigma} \leq$ $\max \left\{\min _{j \in J_{E}}\left\{p_{j}\right\}, \min _{j \in J_{T} \cap J_{1}}\left\{p_{j}\right\}\right\}$ if $t_{s}>d$.

\section{Mixed Integer Linear Programming Methods}

This section presents four distinct ways of formulating the problem $1, h_{1}\left|d_{j}=d\right| \sum_{j=1}^{n}\left(E_{j}+T_{j}\right)$ using mixed integer linear programming. Following is the notation for various indices and parameters used in the models.

Indices

$i$ : index of available time windows,

$j$ : index of jobs,

$k$ : index of sequence positions,

$t$ : index of time periods. 


\section{Parameters}

$n$ : total number of jobs,

$d$ : common due date,

$p_{j}$ : processing time of job $j$,

$D_{i}$ : start time of available time window $\delta_{i}$,

$R_{i}$ : finish time of available time window $\delta_{i}$,

$u b C^{*}$ : an upper bound on the makespan, $u b C^{*}=$ $\max \left\{d, t_{e}\right\}+\sum_{j=1}^{n} p_{j}$,

$M:$ an appropriately large positive number.

3.1. Time-Indexed Decisions on Processing Periods. First, the time-indexed variables on processing periods are considered. The rationale for this approach is to decompose the scheduling horizon into individual periods, where period $t$ starts at time $t$ and ends at time $t+1\left(t \in\left[0, u b C^{*}-1\right]\right)$. Then, the scheduling problem can be regarded as the assignment of unit job segments to unit periods. Let the binary variables $x_{j t}$ equal 1 if job $j$ is processed during period $t \in \Psi$ and 0 otherwise, where $\Psi=\bigcup_{i}\left(\delta_{i} \backslash\left\{R_{i}\right\}\right)$ represents the set of time units in which the machine is available. The following formulation is originally proposed in Low et al. [41]. A major drawback of this formulation is its size. The preliminary tests show that only instances with about 20 jobs can be solved by ILOG CPLEX 12.4. Therefore, a more sophisticated formulation must be considered.

MILP1. Consider

$$
\operatorname{Min} \sum_{j=1}^{n}\left(E_{j}+T_{j}\right)
$$

subject to $\sum_{t \in \Psi} x_{j t}=p_{j}, \quad j=1, \ldots, n$,

$t \cdot x_{j t} \leq C_{j}-1, \quad j=1, \ldots, n ; t \in \Psi$,

$C_{j}-p_{j} \leq t+u b C^{*} \cdot\left(1-x_{j t}\right)$,

$$
j=1, \ldots, n ; t \in \Psi
$$

$$
\begin{aligned}
& \sum_{j=1}^{n} x_{j t} \leq 1, \quad t \in \Psi, \\
& C_{j}-d=T_{j}-E_{j}, \quad j=1, \ldots, n, \\
& x_{j t} \in\{0,1\}, \quad j=1, \ldots, n ; t \in \Psi, \\
& C_{j}, E_{j}, T_{j} \geq 0, \quad j=1, \ldots, n .
\end{aligned}
$$

Constraint (2) enforces that all processing occurs only within set $\Psi$. Constraints (3) and (4) indicate that each job $j$ is processed during periods $C_{j}-p_{j}$ and $C_{j}-1$. Constraint (5) guarantees that at any given period at most one job can be handled. Constraint (6) determines the job earliness and tardiness. This model includes $2 n+(2 n+1)|\Psi|$ constraints, $n|\Psi|$ binary variables, and $3 n$ standard variables.
3.2. Time-Indexed Decisions on Start Times. An alternative approach relies on time-indexed variables on start times. This formulation assumes the same time-discretization as for MILP1. Define $\theta_{i j}=\left\{t \mid D_{i} \leq t \leq R_{i}-p_{j}\right\}$ as the set of time units in which job $j$ is allowed to start its processing in available time window $\delta_{i}$, and define est $t_{j}(t)=\{s \mid s \in$ $\left.\Psi, \max \left\{0, t-p_{j}+1\right\} \leq s \leq t\right\}$ as the set of start times for which job $j$ would be in process in period $t$. Let the binary variables $y_{j t}$ equal 1 if job $j$ starts at period $t \in \Psi$ and 0 otherwise. The formulation is described as follows.

MILP2. Consider

$$
\operatorname{Min} \sum_{j=1}^{n}\left(E_{j}+T_{j}\right)
$$

$$
\begin{aligned}
& \text { subject to } \sum_{t \in \bigcup_{i} \theta_{i j}} y_{j t}=1+|\Psi| \cdot \sum_{t \notin \bigcup_{i} \theta_{i j}} y_{j t}, \\
& \qquad j=1, \ldots, n, \\
& \sum_{j=1}^{n} \sum_{s \in e s t_{j}(t)} y_{j s} \leq 1, \quad t \in \Psi, \\
& \sum_{t \in \bigcup_{i} \theta_{i j}} t \cdot y_{j t}+p_{j}-d=T_{j}-E_{j}, \\
& y_{j t} \in\{0,1\}, \quad j=1, \ldots, n, \forall t \in \Psi, \\
& E_{j}, T_{j} \geq 0, \quad j=1, \ldots, n .
\end{aligned}
$$

Constraint (10) ensures that each job is processed once, where $\bigcup_{i} \theta_{i j}$ represents the set of time units in which job $j$ is allowed to start its processing in the scheduling horizon. Constraint (11) states that at most one job can be handled at any time. Constraint (12) calculates the values of earliness and tardiness of jobs. This model contains $2 n+|\Psi|$ constraints, $n|\Psi|$ binary variables, and $2 n$ standard variables.

3.3. Sequence-Position Decisions. In this formulation, the key binary variables are $x_{i j k}=1$ if job $j$ is assigned to the $k$ th $\left(k=1, \ldots, l_{i}\right)$ position in available time window $\delta_{i}$ and 0 otherwise, where $l_{i}$ is the maximum number of jobs that can be scheduled in $\delta_{i}$. It is obtained easily by inserting the shortest jobs. Define $C T_{i k}, E_{i k}$, and $T_{i k}$ as the completion time, earliness, and tardiness of the job scheduled $k$ th in available time window $\delta_{i}$, respectively. From Property 1, it is sufficient to consider only schedules in which jobs are contiguous in each available time window. Nevertheless, an optimal schedule may not start processing the jobs immediately at the beginning of each available time window. Thus, the variables $S T_{i}$ are introduced to represent the start time of the first job processed in available time window $\delta_{i}$. With the above notation the problem can be modelled as follows. 
MILP3. Consider

$$
\operatorname{Min} \sum_{i=1}^{2} \sum_{k=1}^{l_{i}}\left(E_{i k}+T_{i k}\right)
$$

subject to $\sum_{i=1}^{2} \sum_{k=1}^{l_{i}} x_{i j k}=1, \quad j=1, \ldots, n$,

$$
\begin{aligned}
& \sum_{j=1}^{n} x_{i j k} \leq 1, \quad i=1,2 ; k=1, \ldots, l_{i}, \\
& C T_{i k}=S T_{i}+\sum_{j=1}^{n} \sum_{s=1}^{k} p_{j} \cdot x_{i j s}, \\
& i=1,2 ; k=1, \ldots, l_{i},
\end{aligned}
$$

$C T_{i k}-d$

$$
\begin{aligned}
\leq T_{i k}-E_{i k}+u b C^{*} & \left(1-\sum_{j=1}^{n} x_{i j k}\right), \\
i & =1,2 ; k=1, \ldots, l_{i},
\end{aligned}
$$

$$
d-C T_{i k}
$$$$
\leq E_{i k}-T_{i k}+u b C^{*} \cdot\left(1-\sum_{j=1}^{n} x_{i j k}\right),
$$

$$
i=1,2 ; k=1, \ldots, l_{i} \text {, }
$$$$
E_{i k}+T_{i k} \leq u b C^{*} \cdot \sum_{j=1}^{n} x_{i j k}
$$

$$
i=1,2 ; k=1, \ldots, l_{i},
$$

$$
C T_{i k} \leq R_{i}, \quad i=1,2 ; k=1, \ldots, l_{i},
$$

$S T_{i} \geq D_{i}, \quad i=1,2$,

$x_{i j k} \in\{0,1\}$,

$$
i=1,2 ; j=1, \ldots, n ; k=1, \ldots, l_{i},
$$

$S T_{i} \geq 0, \quad i=1,2$,

$$
C T_{i k}, E_{i k}, T_{i k} \geq 0 \text {, }
$$

$$
i=1,2 ; k=1, \ldots, l_{i} .
$$

Constraint (16) indicates that each job is assigned to exactly one position in one available time window. Constraint (17) guarantees the assignment of at most one job to each position in each available time window. Constraint (18) gives the completion time of the job that is assigned to the $k$ th position in $\delta_{i}$. Constraints (19)-(21) measure the deviation of each job's completion time from the common due date. If there exists a job that is scheduled in the $k$ th position in $\delta_{i}$ (i.e., $\sum_{j=1}^{n} x_{i j k}=1$ ), then its earliness and tardiness can be determined by equation $C T_{i k}-d=T_{i k}-E_{i k}$, which follows directly from Constraints (19) and (20). Otherwise, Constraint (21) enforces $E_{i k}$ and $T_{i k}$ equal to zero. Constraints (22) and (23) concern the machine unavailability. This model includes $n+6 \sum_{i=1}^{2} l_{i}+2$ constraints (i.e., $13 n+2$ at most), $n \sum_{i=1}^{2} l_{i}$ binary variables (i.e., $2 n^{2}$ at most), and $3 \sum_{i=1}^{2} l_{i}+2$ standard variables (i.e., $6 n+2$ at most).

3.4. Precedence Decisions. This formulation is based on the precedence variables. Let $x_{i j^{\prime} j}$ be the binary variables equal to 1 if job $j^{\prime}$ precedes job $j$ in available time window $\delta_{i}$, and let $y_{i j}$ be the binary variables equal to 1 if job $j$ is scheduled in available time window $\delta_{i}$. Note that job $j^{\prime}$ is not necessarily positioned immediately before job $j$ in $\delta_{i}$ when $x_{i j^{\prime} j}=1$. Hence, the problem can be modelled as follows.

MILP4. Consider

$$
\begin{aligned}
\text { Min } & \sum_{j=1}^{n}\left(E_{j}+T_{j}\right) \\
\text { subject to } & \sum_{i=1}^{2} y_{i j}=1, \quad j=1, \ldots, n, \\
& 2 \cdot\left(x_{i j^{\prime} j}+x_{i j j^{\prime}}\right) \leq y_{i j}+y_{i j^{\prime}}, \\
& i=1,2 ; j, j^{\prime}=1, \ldots, n ; j^{\prime}<j, \\
& 1+x_{i j^{\prime} j}+x_{i j j^{\prime}} \geq y_{i j}+y_{i j^{\prime}}, \\
& i=1,2 ; j, j^{\prime}=1, \ldots, n ; j^{\prime}<j, \\
& C_{j} \geq C_{j^{\prime}}+p_{j}-M \cdot\left(1-\sum_{i=1}^{2} x_{i j^{\prime} j}\right), \\
& C_{j i}, E_{j}, T_{j} \geq 0, \quad j=1, \ldots, n . \\
& x_{i j^{\prime} j} \in\{0,1\}, \quad j, j^{\prime}=1, \ldots, n, j \neq j^{\prime}, \\
& C_{j} \leq \sum_{i=1}^{2} y_{i j} \cdot R_{i}, \quad j=1, \ldots, n, \\
& \\
& \\
& \\
& \\
& \\
&
\end{aligned}
$$

Constraint (28) states that each job is assigned to exactly one available time window. Constraints (29) and (30) establish the relation between precedence and assignment variables. If jobs $j^{\prime}$ and $j$ are both processed within $\delta_{i}$ (i.e., $y_{i j}=y_{i j^{\prime}}=1$ ), then job $j^{\prime}$ is scheduled either before or after job $j$ in $\delta_{i}$; that is, $x_{i j^{\prime} j}+x_{i j j^{\prime}}=1$. Otherwise, there is no need 
to consider the precedence relationship between jobs $j^{\prime}$ and $j$ in $\delta_{i}$ (i.e., $x_{i j^{\prime} j}=x_{i j j^{\prime}}=0$ ). Constraint (31) computes for every job $j \neq j^{\prime}$ the completion time when job $j^{\prime}$ precedes job $j$ in the same available time window. Otherwise, the constraint is redundant. Constraints (32) and (33) describe the limits of the machine availabilities. Constraint (34) defines the earliness and tardiness of jobs. This model contains $3 n^{2}+n$ constraints, $2 n^{2}$ binary variables, and $3 n$ standard variables.

Unlike the other formulations, it can be seen that the sizes of the time-indexed formulations (MILP1 and MILP2) depend on the length of the scheduling horizon. This can lead to models with quite a large number of binary variables; however, the time-indexed formulations may be efficient in other respects. More specifically, tightening the linear relaxation of the formulation plays a key role in convergence of the solution procedure, and it strictly relies on the choice of decision variables used and the relevant constraint structures. To that end, the performance of these above formulations will be evaluated in computational study.

\section{Dynamic Programming Based Method}

In this section, a dynamic programming model for the problem $1, h_{1}\left|d_{j}=d\right| \sum_{j=1}^{n}\left(E_{j}+T_{j}\right)$ is presented. This method is an extension of Ventura and Weng's dynamic programming [13]. Here, we show that it can be applied with slight modification to the case with an unavailability interval.

Since the machine is unavailable for processing during the time interval $\left[t_{s}, t_{e}\right]$, there are three cases to consider: (1) $t_{e}<$ $d$, (2) $t_{s}>d$, and (3) $t_{s} \leq d \leq t_{e}$. The algorithms are developed below separately for each case. Assume for convenience that the jobs are renumbered in the order defined for SPT.

Algorithm for Case $1\left(t_{e}<d\right)$. Let $f(j, t, s)$ denote the minimum cost for the $j$-jobs partial problem, given that the total processing time of the jobs in $\delta_{1}$ is $t$, and the earliest job in $\delta_{2}$ starts at time $s$. For $j=1, \ldots, n, t=$ $0, \ldots, \min \left\{t_{s}, \sum_{h=1}^{j} p_{h}\right\}$ and $s=t_{e}, \ldots, d+p_{n}-1$, the recursive three-term equation is defined as (41), in which the first two terms represent the value of $f(j, t, s)$ if job $j$ is scheduled after $t_{e}$ and the last term represents the value of $f(j, t, s)$ if job $j$ is scheduled before $t_{s}$. From Properties 4 and 5, if $s<d$ then at the stage of scheduling job $j$ in $\delta_{2}$ two decisions should be considered: job $j$ can be scheduled as the first job, starting at time $s$, or as the last one, then starting at time $s+\sum_{h=1}^{j-1} p_{h}-t$. The first decision leads to the partial schedule presented in Figure 1. The cost of this partial schedule can be calculated according to

$$
f\left(j-1, t, s+p_{j}\right)+\left|s+p_{j}-d\right| .
$$

The second decision results in the partial schedule shown in Figure 2, and its cost is given by

$$
f(j-1, t, s)+\left|s+\sum_{h=1}^{j} p_{h}-t-d\right| .
$$

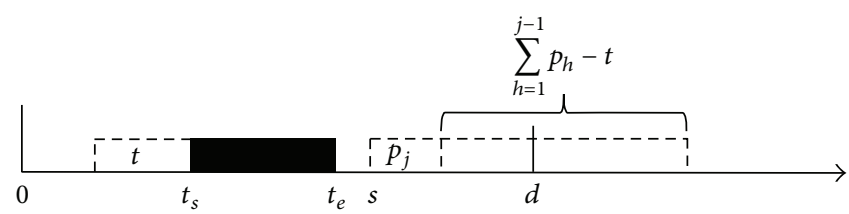

Figure 1: A partial schedule in $\delta_{2}$ with job $j$ starting at time $s$.

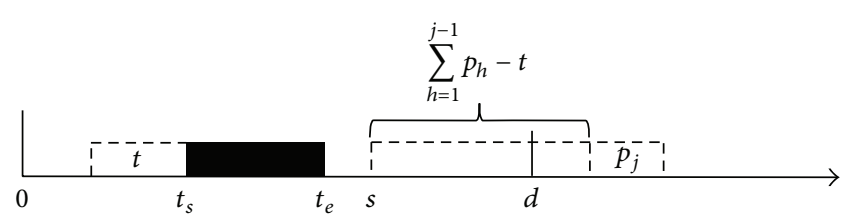

Figure 2: A partial schedule in $\delta_{2}$ with job $j$ starting at time $s+$ $\sum_{h=1}^{j-1} p_{h}-t$.

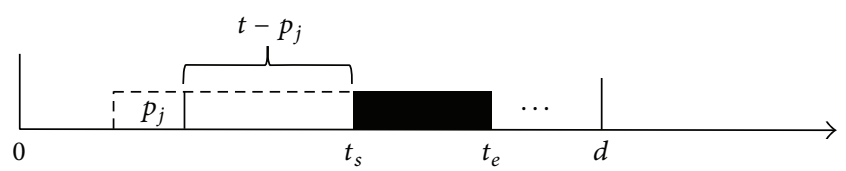

Figure 3: A partial schedule in $\delta_{1}$ with job $j$ starting at time $t_{s}-t$.

If $s \geq d$ then all jobs in $\delta_{2}$ are tardy, so job $j$ has to be scheduled as the last one in $\delta_{2}$. As a result, in this situation the first term in recursion can be omitted.

$f\left(j-1, t-p_{j}, s\right)+d-t_{s}+t-p_{j}$ is the value of $f(j, t, s)$ if job $j$ is scheduled in $\delta_{1}$. By Properties 3 and 4 , the partial schedule in $\delta_{1}$ is constructed in a backward manner such that the last job in $\delta_{1}$ finishes exactly at time $t_{s}$ (see Figure 3 ).

Initial Condition. The initial condition is

$$
f(0, t, s)= \begin{cases}0 & \text { if } t=0 \\ +\infty & \text { otherwise }\end{cases}
$$

The Recursion. The recursion is

$$
\begin{aligned}
& f(j, t, s) \\
& =\min \left\{\begin{array}{l}
f\left(j-1, t, s+p_{j}\right)+\left|s+p_{j}-d\right|, \\
f(j-1, t, s)+\left|s+\sum_{h=1}^{j} p_{h}-t-d\right|, \\
f\left(j-1, t-p_{j}, s\right)+d-t_{s}+t-p_{j},
\end{array}\right.
\end{aligned}
$$

where $f\left(j-1, t, s+p_{j}\right)+\left|s+p_{j}-d\right|=f(j-1, t, s)+$ $\left|s+\sum_{h=1}^{j} p_{h}-t-d\right|=+\infty$ if $t>\min \left\{t_{s}, \sum_{h=1}^{j-1} p_{h}\right\}$ and $f\left(j-1, t-p_{j}, s\right)+d-t_{s}+t-p_{j}=+\infty$ if $t-p_{j}<0$. The optimal objective value is equal to $\min _{0 \leq t \leq \min \left\{t_{s}, \sum_{j=1}^{n} p_{j}\right\}, t_{e} \leq s \leq d} f(n, t, s)$. For each state $(j, t, s)$, there are at most three operations. It follows from Property 5 that if job 1 starts after $d$ in an optimal schedule, it must start on or before $d+p_{n}-1$, which implies that $s \leq d+p_{n}-1$. In addition, the value of the state variable $t$ is bounded by $t_{s}$. Therefore, the time complexity of the algorithm is $\mathrm{O}\left(n t_{s}\left(d+p_{n}-t_{e}\right)\right)$. 


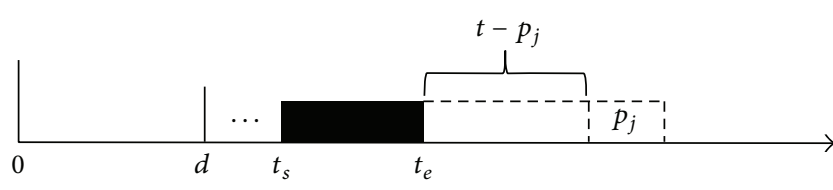

FIGURE 4: A partial schedule in $\delta_{2}$ with job $j$ starting at time $t_{e}+t-p_{j}$.

Algorithm for Case $2\left(t_{s}>d\right)$. Following the same reasoning discussed in Case 1 , let $f(j, s, t)$ denote the minimum cost for the $j$-jobs partial problem, given that the earliest job in $\delta_{1}$ starts at time $s$, and the total processing time of the jobs in $\delta_{2}$ is $t$. For $j=1, \ldots, n, s=0, \ldots, \min \left\{t_{s}, d+p_{n}-1\right\}$ and $t=0, \ldots, \sum_{h=1}^{j} p_{h}$, the recursive equation takes the following form.

Initial Condition. The initial condition is

$$
f(0, s, t)= \begin{cases}0 & \text { if } t=0 \\ +\infty & \text { otherwise }\end{cases}
$$

The Recursion. The recursion is

$$
\begin{array}{r}
f(j, s, t) \\
=\min \left\{\begin{array}{l}
f\left(j-1, s+p_{j}, t\right)+\left|s+p_{j}-d\right|, \\
f(j-1, s, t)+\left|s+\sum_{h=1}^{j} p_{h}-t-d\right|, \\
f\left(j-1, s, t-p_{j}\right)+t_{e}+t-d,
\end{array}\right.
\end{array}
$$

where $f\left(j-1, s+p_{j}, t\right)+\left|s+p_{j}-d\right|=+\infty$ if $s>$ $\min \left\{t_{s}+t-\sum_{h=1}^{j} p_{h}, \min \left\{t_{s}, d+p_{n}-1\right\}-p_{j}\right\}$ or $t>\sum_{h=1}^{j-1} p_{h}$, $f(j-1, s, t)+\left|s+\sum_{h=1}^{j} p_{h}-t-d\right|=+\infty$ if $s>t_{s}+t-\sum_{h=1}^{j} p_{h}$ or $t>\sum_{h=1}^{j-1} p_{h}$, and $f\left(j-1, s, t-p_{j}\right)+t_{e}+t-d=+\infty$ if $t-p_{j}<0$. Clearly if $s \geq d$, it is sufficient from Property 4 to consider that job $j$ is scheduled either as the last one in $\delta_{1}$, starting at time $s+\sum_{h=1}^{j-1} p_{h}-t$, or as the last one in $\delta_{2}$, then starting at time $t_{e}+$ $t-p_{j}$ (see Figure 4). Therefore, in this situation the first term in recursion can be eliminated. The optimal objective value is found for $\min _{0 \leq s \leq \min \left\{t_{s}, d+p_{n}-1\right\}, 0 \leq t \leq \sum_{j=1}^{n} p_{j}} f(n, s, t)$. Each state $(j, s, t)$ requires at most three operations, and the state variables $s$ and $t$ take $t_{s}$ and $\sum_{j=1}^{n} p_{j}$ as their maximum possible values, respectively. Hence, the overall time complexity of the algorithm is bounded by $\mathrm{O}\left(n t_{s}\left(\sum_{j=1}^{n} p_{j}\right)\right)$.

Algorithm for Case $3\left(t_{s} \leq d \leq t_{e}\right)$. In this case, the problem is reduced to determining the optimal assignment of the jobs to the available time windows. Once the membership in the two available time windows is specified, the optimal schedules in each available time window can be found by Properties 1, 3, and 4 . Let $f(j, t)$ be the minimum cost for the $j$-jobs partial problem, where the total processing time of the jobs in $\delta_{1}$ is $t$. For $j=1, \ldots, n$ and $t=0, \ldots, \min \left\{t_{s}, \sum_{h=1}^{j} p_{h}\right\}$, the recursive two-term equation is defined as (45), in which the first term represents the value of $f(j, t)$ if job $j$ is scheduled in $\delta_{1}$ and the second term represents the value of $f(j, t)$ if job $j$ is scheduled in $\delta_{2}$.

Initial Condition. The initial condition is

$$
f(0, t)= \begin{cases}0 & \text { if } t=0 \\ +\infty & \text { otherwise }\end{cases}
$$

The Recursion. The recursion is

$$
f(j, t)=\min \left\{\begin{array}{l}
f\left(j-1, t-p_{j}\right)+d-t_{s}+t-p_{j}, \\
f(j-1, t)+t_{e}+\sum_{h=1}^{j} p_{h}-t-d,
\end{array}\right.
$$

where $f\left(j-1, t-p_{j}\right)+d-t_{s}+t-p_{j}=+\infty$ if $t-p_{j}<0$ and $f(j-1, t)+t_{e}+\sum_{h=1}^{j} p_{h}-t-d=+\infty$ if $t>\min \left\{t_{s}, \sum_{h=1}^{j-1} p_{h}\right\}$. The optimal objective value is equal to $\min _{0 \leq t \leq \min \left\{t_{s}, \sum_{j=1}^{n} p_{j}\right\}} f(n, t)$. As described above, it can be established that the algorithm finds an optimal schedule in $\mathrm{O}\left(n t_{s}\right)$ time.

\section{Experimental Results}

In this section, the numerical results are provided to assess the performance of the different methods presented above. The dynamic programming algorithms were written in $\mathrm{C}++$ language and the mixed integer linear programs were solved by ILOG CPLEX 12.4. The experiments were conducted on a PC with a $3.4 \mathrm{GHz}$ processor and $4 \mathrm{~GB}$ RAM.

5.1. Comparison between the Proposed Methods. Here, the computation times (in CPU seconds) and the optimality gaps of the proposed methods are compared. The problem instances were generated as follows:

(1) The number of jobs was chosen from 5 to 200.

(2) The job processing times followed the discrete uniform distribution [1, $\left.p_{\max }=10\right]$.

(3) The start time of the unavailability interval was set at $\left\lfloor P \sum_{j=1}^{n} p_{j}\right\rfloor$ with the proportionality coefficient $P \in$ $\{0.25,0.5,0.75\}$. For simplicity, the duration of the unavailability interval was set to be $\left[\bar{p}=\left(\sum_{j=1}^{n} p_{j}\right) / n\right\rfloor$. The integer due date was generated uniformly as follows.

Case $1\left(t_{e}<d\right)$. Consider

$$
d \in\left[t_{e}+\frac{\sum_{j=1}^{n} p_{j}}{3}(1-R), t_{e}+\frac{\sum_{j=1}^{n} p_{j}}{3}(1+R)\right] .
$$

Case $2\left(t_{s}>d\right)$. Consider

$$
d \in\left[\frac{t_{s}}{2}(1-R), \frac{t_{s}}{2}(1+R)\right] .
$$

Case $3\left(t_{s} \leq d \leq t_{e}\right)$. Consider

$$
d \in\left[\frac{t_{s}+t_{e}-R \bar{p}}{2}, \frac{t_{s}+t_{e}+R \bar{p}}{2}\right],
$$


TABLE 1: Comparison between the exact methods.

\begin{tabular}{|c|c|c|c|c|c|c|c|c|c|c|c|}
\hline \multirow{2}{*}{ Case } & \multirow{2}{*}{$n$} & \multicolumn{2}{|c|}{ MILP1 } & \multicolumn{2}{|c|}{ MILP2 } & \multicolumn{2}{|c|}{ MILP3 } & \multicolumn{2}{|c|}{ MILP4 } & \multicolumn{2}{|c|}{$\mathrm{DP}$} \\
\hline & & Time (s) & GAP (\%) & Time (s) & GAP (\%) & Time (s) & GAP (\%) & Time (s) & GAP (\%) & Time (s) & GAP (\%) \\
\hline \multirow{12}{*}{1} & 5 & 2.31 & 0.00 & 1.56 & 0.00 & 2.09 & 0.00 & 2.52 & 0.00 & $<0.01$ & 0.00 \\
\hline & 10 & 147.25 & 0.00 & 3.13 & 0.00 & 6.03 & 0.00 & 23.78 & 0.00 & $<0.01$ & 0.00 \\
\hline & 15 & 851.74 & $42.17^{*}$ & 2.81 & 0.00 & 30.39 & 0.00 & 3819.94 & 0.30 & $<0.01$ & 0.00 \\
\hline & 20 & o. o. m. & - & 3.23 & 0.00 & 44.95 & 0.00 & 5000.00 & $2.04^{*}$ & $<0.01$ & 0.00 \\
\hline & 25 & o. o. m. & - & 4.67 & 0.00 & 3012.04 & $0.26^{*}$ & 5000.00 & $3.79^{*}$ & $<0.01$ & 0.00 \\
\hline & 30 & o. o. m. & - & 7.35 & 0.00 & 4311.25 & $0.49^{*}$ & 5000.00 & $6.42^{*}$ & $<0.01$ & 0.00 \\
\hline & 35 & o. o. m. & - & 17.46 & 0.00 & o. o. m. & - & o. o. m. & $15.93^{*}$ & $<0.01$ & 0.00 \\
\hline & 50 & o. o. m. & - & 63.72 & 0.00 & o. o. m. & - & o. o. m. & $58.15^{*}$ & $<0.01$ & 0.00 \\
\hline & 75 & o. o. m. & - & 86.54 & 0.00 & o. o. m. & - & o. o. m. & - & 0.02 & 0.00 \\
\hline & 100 & o. o. m. & - & 303.81 & 0.00 & o. o. m. & - & o. o. m. & - & 0.02 & 0.00 \\
\hline & 150 & o. o. m. & - & 2138.73 & 0.00 & o. o. m. & - & o. o. m. & - & 0.05 & 0.00 \\
\hline & 200 & o. o. m. & - & 5000.00 & $1.56^{*}$ & o. o. m. & - & o. o. m. & - & 0.06 & 0.00 \\
\hline \multirow{12}{*}{2} & 5 & 5.49 & 0.00 & 1.78 & 0.00 & 2.83 & 0.00 & 2.45 & 0.00 & $<0.01$ & 0.00 \\
\hline & 10 & 546.57 & 0.00 & 4.06 & 0.00 & 5.57 & 0.00 & 6.89 & 0.00 & $<0.01$ & 0.00 \\
\hline & 15 & 2741.33 & $27.96^{*}$ & 3.76 & 0.00 & 7.04 & 0.00 & 184.47 & 0.00 & $<0.01$ & 0.00 \\
\hline & 20 & o. o. m. & $41.58^{*}$ & 5.62 & 0.00 & 21.37 & 0.00 & 5000.00 & $0.43^{*}$ & $<0.01$ & 0.00 \\
\hline & 25 & o. o. m. & - & 22.21 & 0.00 & 2721.91 & $0.11^{*}$ & 5000.00 & $2.54^{*}$ & $<0.01$ & 0.00 \\
\hline & 30 & o. o. m. & - & 46.47 & 0.00 & 3520.68 & $0.09^{*}$ & 5000.00 & $11.46^{*}$ & $<0.01$ & 0.00 \\
\hline & 35 & o. o. m. & - & 194.71 & 0.00 & o. o. m. & - & o. o. m. & $15.87^{*}$ & $<0.01$ & 0.00 \\
\hline & 50 & o. o. m. & - & 225.36 & 0.00 & o. o. m. & - & o. o. m. & $47.63^{*}$ & 0.01 & 0.00 \\
\hline & 75 & o. o. m. & - & 473.98 & 0.00 & o. o. m. & - & o. o. m. & - & 0.03 & 0.00 \\
\hline & 100 & o. o. m. & - & 637.14 & 0.00 & o. o. m. & - & o. o. m. & - & 0.05 & 0.00 \\
\hline & 150 & o. o. m. & - & 2361.50 & 0.00 & o. o. m. & - & o. o. m. & - & 0.11 & 0.00 \\
\hline & 200 & o. o. m. & - & 5000.00 & $1.18^{*}$ & o. o. m. & - & o. o. m. & - & 0.24 & 0.00 \\
\hline \multirow{12}{*}{3} & 5 & 7.27 & 0.00 & 1.48 & 0.00 & 3.83 & 0.00 & 10.98 & 0.00 & $<0.01$ & 0.00 \\
\hline & 10 & 403.95 & 0.00 & 2.80 & 0.00 & 9.17 & 0.00 & 835.60 & 0.00 & $<0.01$ & 0.00 \\
\hline & 15 & o. o. m. & $22.81^{*}$ & 2.93 & 0.00 & 66.31 & 0.00 & 5000.00 & $0.03^{*}$ & $<0.01$ & 0.00 \\
\hline & 20 & o. o. m. & - & 3.85 & 0.00 & 2206.94 & $0.25^{*}$ & 5000.00 & $0.12^{*}$ & $<0.01$ & 0.00 \\
\hline & 25 & o. o. m. & - & 4.87 & 0.00 & 3136.89 & $0.30^{*}$ & 5000.00 & $1.96^{*}$ & $<0.01$ & 0.00 \\
\hline & 30 & o. o. m. & - & 12.63 & 0.00 & o. o. m. & - & 5000.00 & $1.50^{*}$ & $<0.01$ & 0.00 \\
\hline & 35 & o. o. m. & - & 24.38 & 0.00 & o. o. m. & - & o. o. m. & $4.32^{*}$ & $<0.01$ & 0.00 \\
\hline & 50 & o. o. m. & - & 67.50 & 0.00 & o. o. m. & - & o. o. m. & $26.35^{*}$ & $<0.01$ & 0.00 \\
\hline & 75 & o. o. m. & - & 93.79 & 0.00 & o. o. m. & - & o. o. m. & - & $<0.01$ & 0.00 \\
\hline & 100 & o. o. m. & - & 235.18 & 0.00 & o. o. m. & - & o. o. m. & - & $<0.01$ & 0.00 \\
\hline & 150 & o. o. m. & - & 2977.05 & 0.00 & o. o. m. & - & o. o. m. & - & $<0.01$ & 0.00 \\
\hline & 200 & o. o. m. & - & 5000.00 & $0.42^{*}$ & o. o. m. & - & o. o. m. & - & $<0.01$ & 0.00 \\
\hline
\end{tabular}

$\mathrm{DP}=$ dynamic programming; $\mathrm{o}$. o. $\mathrm{m}$. = out of memory.

of which the due date factor was chosen from $R \in$ $\{0.3,0.5,0.7\}$.

For each case, 10 instances were randomly generated for each problem size. The results of this experiment are summarized in Table 1. The symbol "-_" denotes the cases that no feasible solution found within a time limit 5000 seconds or the program is interrupted due to lack of memory. The symbol " $*$ " denotes that the optimality gap is calculated as $[(C(\mathrm{IB})-$ optimum)/optimum $] \times 100 \%$, where $C(\mathrm{IB})$ is the incumbent solution obtained before the program is terminated.

According to these results, it is clear that MILP2 and the dynamic programming method are much more effective than the other mixed integer linear programming formulations. MILP2 was able to handle the problems with close to 150 jobs, while it was difficult to use the latter approaches to solve instances with more than 20 jobs. However, the obtained results corroborate the findings in Kacem et al. [44] that the dynamic programming method could be superior to other 
TABLE 2: Dynamic programming method performance.

\begin{tabular}{|c|c|c|c|c|}
\hline Case & $n$ & $\begin{array}{c}p_{\max }=50 \\
\text { Time }(\mathrm{s})\end{array}$ & $\begin{array}{c}p_{\max }=100 \\
\text { Time }(\mathrm{s})\end{array}$ & $\begin{array}{c}p_{\max }=200 \\
\text { Time }(\mathrm{s})\end{array}$ \\
\hline \multirow{12}{*}{1} & 5 & $<0.01$ & $<0.01$ & $<0.01$ \\
\hline & 10 & $<0.01$ & 0.01 & 0.03 \\
\hline & 15 & 0.01 & 0.02 & 0.09 \\
\hline & 20 & 0.01 & 0.04 & 0.18 \\
\hline & 25 & 0.02 & 0.09 & 0.29 \\
\hline & 30 & 0.03 & 0.10 & 0.63 \\
\hline & 35 & 0.05 & 0.23 & 0.83 \\
\hline & 50 & 0.14 & 0.66 & 1.86 \\
\hline & 75 & 0.50 & 3.09 & 7.32 \\
\hline & 100 & 1.44 & 3.82 & 24.16 \\
\hline & 150 & 2.85 & 5.11 & 70.54 \\
\hline & 200 & 6.82 & 7.35 & 177.91 \\
\hline \multirow{12}{*}{2} & 5 & $<0.01$ & $<0.01$ & $<0.01$ \\
\hline & 10 & $<0.01$ & $<0.01$ & 0.02 \\
\hline & 15 & 0.01 & 0.03 & 0.15 \\
\hline & 20 & 0.01 & 0.03 & 0.17 \\
\hline & 25 & 0.02 & 0.08 & 0.22 \\
\hline & 30 & 0.04 & 0.14 & 0.75 \\
\hline & 35 & 0.06 & 0.25 & 0.88 \\
\hline & 50 & 0.20 & 0.67 & 1.55 \\
\hline & 75 & 0.58 & 3.25 & 6.81 \\
\hline & 100 & 1.23 & 5.19 & 19.73 \\
\hline & 150 & 3.85 & 7.96 & 58.62 \\
\hline & 200 & 9.76 & 11.25 & 183.36 \\
\hline \multirow{12}{*}{3} & 5 & $<0.01$ & 0.01 & $<0.01$ \\
\hline & 10 & $<0.01$ & $<0.01$ & $<0.01$ \\
\hline & 15 & $<0.01$ & $<0.01$ & $<0.01$ \\
\hline & 20 & $<0.01$ & $<0.01$ & $<0.01$ \\
\hline & 25 & $<0.01$ & $<0.01$ & $<0.01$ \\
\hline & 30 & $<0.01$ & $<0.01$ & $<0.01$ \\
\hline & 35 & $<0.01$ & $<0.01$ & $<0.01$ \\
\hline & 50 & $<0.01$ & $<0.01$ & $<0.01$ \\
\hline & 75 & $<0.01$ & 0.01 & $<0.01$ \\
\hline & 100 & $<0.01$ & 0.01 & 0.02 \\
\hline & 150 & $<0.01$ & 0.01 & 0.02 \\
\hline & 200 & $<0.01$ & 0.01 & 0.02 \\
\hline
\end{tabular}

methods. As compared to MILP2, the dynamic programming method was more efficient in attaining the optimal schedules and no problems due to memory requirement. In addition, the superiority of the dynamic programming method became more significant as the problem size increased.

The dynamic programming method is complex. So, we add particularly further experiments with $p_{\max }=50,100$, and 200 to evaluate the impact of the length of the scheduling horizon on the method. Table 2 presents computation times required to obtain the optimal solutions for each case. As the results show, the dynamic programming method became relatively expensive, in terms of CPU time, when the value of $\sum_{j=1}^{n} p_{j}$ increased. Moreover, as compared to the results of the mixed integer linear programming methods for the problems with $p_{\max }=10$, the dynamic programming method was still capable of quickly solving problems with hundreds of jobs. Given the above observations, we can conclude that the dynamic programming method may be more promising for the addressed problem.

5.2. Dynamic Programming Method Experiments. In this section, the influence of the proportionality coefficient $P$ and the due date factor $R$ on the performance of the dynamic programming method is analyzed. The number of jobs varied in $n \in\{100,300,500,700,1000\}$, and the job processing times followed a discrete uniform distribution defined by the interval $[1,20]$. The due date, start time, and the duration of the unavailability interval were set as those described in the previous experiment. For each treble $(n, P, R), 10$ instances were randomly generated. Hence, this experiment contains a total of 450 instances for each case. For Cases 1 and 2, it can be observed from Table 3 that for the same problem sizes the average of CPU times became higher when the value of $P$ increased. The results also indicate that as compared to Case 1 when $P$ was small $(\leq 0.5)$ the problems of Case 2 were relatively easy to solve. Nevertheless, it is noticed that the variation of $R$ did not have a significant influence on the problem hardness. With respect to Case 3, the results indicate that for the same problem sizes the larger the value of $P$ was the harder the problems became. It seems that the value of $R$ had no effect on the performance of this method. Indeed, in this case the algorithm is implemented in $\mathrm{O}\left(n t_{s}\right)$ time and therefore, its complexity is independent of the due date setting.

\section{Conclusions}

This paper addresses the single-machine scheduling problem to minimize the TET in relation to a common due date, where the machine is subject to a given unavailability interval during which the production is not allowed. To the best of our knowledge, no exact algorithms have previously been published for this problem. Therefore, this study proposes solution methodologies and properties of an optimal schedule for the purpose of exposing insights that may ultimately be useful in research on more complicated models.

First, four mixed integer linear programming formulations with different types of binary variables that seize the scheduling decisions are introduced. Observing that these formulations are time-consuming to derive an optimal solution with the increase of the number of jobs, a dynamic programming method based on the solution properties is then proposed to deal with large-scale problems. Numerical results for problems with up to 1000 jobs demonstrate that the dynamic programming method is well suited to finding optimal schedules in comparison with the mixed integer linear programming methods and its advantage gets bigger as the problems size increases. In summary, when the complexity of the dynamic programming method remains correct, it is very promising for practical problems. 
TABLE 3: Influence of the proportionality coefficient $P$ and the due date factor $R$ on the dynamic programming method.

\begin{tabular}{|c|c|c|c|c|}
\hline$(P, R)$ & $n$ & $\begin{array}{c}\text { Case } 1 \\
\text { Time (s) }\end{array}$ & $\begin{array}{c}\text { Case } 2 \\
\text { Time (s) }\end{array}$ & $\begin{array}{c}\text { Case } 3 \\
\text { Time (s) }\end{array}$ \\
\hline \multirow{5}{*}{$(0.25,0.3)$} & 100 & 0.14 & 0.09 & $<0.01$ \\
\hline & 300 & 3.16 & 2.35 & $<0.01$ \\
\hline & 500 & 15.03 & 11.23 & 0.01 \\
\hline & 700 & 39.49 & 24.58 & 0.01 \\
\hline & 1000 & 130.87 & 82.76 & 0.03 \\
\hline \multirow{5}{*}{$(0.25,0.5)$} & 100 & 0.09 & 0.08 & $<0.01$ \\
\hline & 300 & 3.08 & 2.36 & $<0.01$ \\
\hline & 500 & 16.69 & 10.13 & $<0.01$ \\
\hline & 700 & 36.46 & 25.28 & 0.01 \\
\hline & 1000 & 134.17 & 79.13 & 0.03 \\
\hline \multirow{5}{*}{$(0.25,0.7)$} & 100 & 0.11 & 0.09 & $<0.01$ \\
\hline & 300 & 3.20 & 2.15 & $<0.01$ \\
\hline & 500 & 16.75 & 11.47 & $<0.01$ \\
\hline & 700 & 45.84 & 27.62 & 0.01 \\
\hline & 1000 & 122.90 & 88.58 & 0.03 \\
\hline \multirow{5}{*}{$(0.5,0.3)$} & 100 & 0.18 & 0.13 & $<0.01$ \\
\hline & 300 & 5.37 & 4.99 & $<0.01$ \\
\hline & 500 & 23.15 & 23.05 & $<0.01$ \\
\hline & 700 & 66.22 & 61.52 & 0.01 \\
\hline & 1000 & 201.46 & 185.01 & 0.05 \\
\hline \multirow{5}{*}{$(0.5,0.5)$} & 100 & 0.18 & 0.24 & $<0.01$ \\
\hline & 300 & 5.25 & 5.17 & $<0.01$ \\
\hline & 500 & 28.75 & 22.25 & $<0.01$ \\
\hline & 700 & 69.43 & 60.68 & 0.02 \\
\hline & 1000 & 224.80 & 166.46 & 0.04 \\
\hline \multirow{5}{*}{$(0.5,0.7)$} & 100 & 0.16 & 0.18 & $<0.01$ \\
\hline & 300 & 5.74 & 4.83 & $<0.01$ \\
\hline & 500 & 25.67 & 24.67 & $<0.01$ \\
\hline & 700 & 71.19 & 64.09 & 0.02 \\
\hline & 1000 & 205.13 & 200.98 & 0.04 \\
\hline \multirow{5}{*}{$(0.75,0.3)$} & 100 & 0.28 & 0.30 & $<0.01$ \\
\hline & 300 & 6.04 & 7.71 & $<0.01$ \\
\hline & 500 & 30.92 & 34.93 & 0.01 \\
\hline & 700 & 82.50 & 93.58 & 0.02 \\
\hline & 1000 & 305.83 & 293.25 & 0.06 \\
\hline \multirow{5}{*}{$(0.75,0.5)$} & 100 & 0.27 & 0.29 & $<0.01$ \\
\hline & 300 & 6.51 & 7.70 & $<0.01$ \\
\hline & 500 & 33.76 & 35.08 & 0.01 \\
\hline & 700 & 79.24 & 100.42 & 0.03 \\
\hline & 1000 & 255.73 & 298.25 & 0.06 \\
\hline \multirow{5}{*}{$(0.75,0.7)$} & 100 & 0.32 & 0.49 & $<0.01$ \\
\hline & 300 & 5.49 & 8.68 & $<0.01$ \\
\hline & 500 & 36.81 & 43.54 & 0.01 \\
\hline & 700 & 90.10 & 116.32 & 0.02 \\
\hline & 1000 & 231.43 & 312.97 & 0.06 \\
\hline
\end{tabular}

Although the computational complexity associated with the mixed integer linear programming formulations makes it usually difficult for optimization software that addresses industrial-dimensioned problems in reasonable solution time, the mixed integer linear programming methods are useful to understand the structure of the problem and may be the better algorithmic choice when convenience in implementation is considered. Further research will be devoted to deriving of valid inequalities and investigation of the development of tighter upper bounds for the total processing time in each available time window in order to improve the computational efficiency of the proposed methods. It might be interesting to extend our schemes to the problem with multiple unavailability intervals.

\section{Competing Interests}

The authors declare that there are no competing interests regarding the publication of this paper.

\section{References}

[1] K. R. Baker and G. D. Scudder, "Sequencing with earliness and tardiness penalties: a review," Operations Research, vol. 38, no. 1, pp. 22-36, 1990.

[2] D. Biskup and M. Feldmann, "Benchmarks for scheduling on a single machine against restrictive and unrestrictive common due dates," Computers and Operations Research, vol. 28, no. 8, pp. 787-801, 2001.

[3] V. Gordon, J.-M. Proth, and C. Chu, "A survey of the stateof-the-art of common due date assignment and scheduling research," European Journal of Operational Research, vol. 139, no. 1, pp. 1-25, 2002.

[4] V. Lauff and F. Werner, "Scheduling with common due date, earliness and tardiness penalties for multimachine problems: a survey," Mathematical and Computer Modelling, vol. 40, no. 5-6, pp. 637-655, 2004.

[5] J. J. Kanet, "Minimizing the average deviation of job completion times about a common due date," Naval Research Logistics Quarterly, vol. 28, no. 4, pp. 643-651, 1981.

[6] U. Bagchi, R. S. Sullivan, and Y.-L. Chang, "Minimizing mean absolute deviation of completion times about a common due date," Naval Research Logistics Quarterly, vol. 33, no. 2, pp. 227240, 1986.

[7] P. S. Sundararaghavan and M. U. Ahmed, "Minimizing the sum of absolute lateness in single-machine and multimachine scheduling," Naval Research Logistics Quarterly, vol. 31, no. 2, pp. 325-333, 1984.

[8] H. Emmons, "Scheduling to a common due date on parallel uniform processors," Naval Research Logistics, vol. 34, no. 6, pp. 803-810, 1987.

[9] N. G. Hall, W. Kubiak, and S. P. Sethi, "Earliness-tardiness scheduling problems, II: deviation of completion times about a restrictive common due date," Operations Research, vol. 39, no. 5, pp. 847-856, 1991.

[10] J. A. Hoogeveen and S. L. van de Velde, "Scheduling around a small common due date," European Journal of Operational Research, vol. 55, no. 2, pp. 237-242, 1991.

[11] J. A. Hoogeveen, H. Oosterhout, and S. L. van de Velde, "New lower and upper bounds for scheduling around a small common due date," Operations Research, vol. 42, no. 1, pp. 102-110, 1994. 
[12] W. Szwarc, "Single-machine scheduling to minimize absolute deviation of completion times from a common due date," Naval Research Logistics, vol. 36, no. 5, pp. 663-673, 1989.

[13] J. A. Ventura and M. X. Weng, "An improved dynamic programming algorithm for the single-machine mean absolute deviation problem with a restrictive common due date," Operations Research Letters, vol. 17, no. 3, pp. 149-152, 1995.

[14] H. Sarper, "Minimizing the sum of absolute deviations about a common due date for the two-machine flow shop problem," Applied Mathematical Modelling, vol. 19, no. 3, pp. 153-161, 1995.

[15] C. S. Sakuraba, D. P. Ronconi, and F. Sourd, "Scheduling in a two-machine flowshop for the minimization of the mean absolute deviation from a common due date," Computers \& Operations Research, vol. 36, no. 1, pp. 60-72, 2009.

[16] C. S. Sung and J. I. Min, "Scheduling in a two-machine flowshop with batch processing machine(s) for earliness/tardiness measure under a common due date," European Journal of Operational Research, vol. 131, no. 1, pp. 95-106, 2001.

[17] W. K. Yeung, C. Oğuz, and T. C. E. Cheng, "Two-stage flowshop earliness and tardiness machine scheduling involving a common due window," International Journal of Production Economics, vol. 90, no. 3, pp. 421-434, 2004.

[18] V. Lauff and F. Werner, "On the complexity and some properties of multi-stage scheduling problems with earliness and tardiness penalties," Computers \& Operations Research, vol. 31, no. 3, pp. 317-345, 2004.

[19] C.-Y. Lee, L. Lei, and M. Pinedo, "Current trends in deterministic scheduling," Annals of Operations Research, vol. 70, pp. 1-41, 1997.

[20] G. Schmidt, "Scheduling with limited machine availability," European Journal of Operational Research, vol. 121, no. 1, pp. 1-15, 2000.

[21] Y. Ma, C. Chu, and C. Zuo, "A survey of scheduling with deterministic machine availability constraints," Computers and Industrial Engineering, vol. 58, no. 2, pp. 199-211, 2010.

[22] C. Low, M. Ji, C.-J. Hsu, and C.-T. Su, "Minimizing the makespan in a single machine scheduling problems with flexible and periodic maintenance," Applied Mathematical Modelling, vol. 34, no. 2, pp. 334-342, 2010.

[23] G. Mosheiov and J. B. Sidney, "Scheduling a deteriorating maintenance activity on a single machine," Journal of the Operational Research Society, vol. 61, no. 5, pp. 882-887, 2010.

[24] C.-1. Zhao and H.-Y. Tang, "Single machine scheduling with general job-dependent aging effect and maintenance activities to minimize makespan," Applied Mathematical Modelling, vol. 34, no. 3, pp. 837-841, 2010.

[25] F. Ángel-Bello, A. Álvarez, J. Pacheco, and I. Martínez, "A single machine scheduling problem with availability constraints and sequence-dependent setup costs," Applied Mathematical Modelling, vol. 35, no. 4, pp. 2041-2050, 2011.

[26] Y. Huo and H. Zhao, "Bicriteria scheduling concerned with makespan and total completion time subject to machine availability constraints," Theoretical Computer Science, vol. 412, no. 12-14, pp. 1081-1091, 2011.

[27] S.-L. Yang, Y. Ma, D.-L. Xu, and J.-B. Yang, "Minimizing total completion time on a single machine with a flexible maintenance activity," Computers and Operations Research, vol. 38, no. 4, pp. 755-770, 2011.

[28] J.-Y. Lee and Y.-D. Kim, "Minimizing the number of tardy jobs in a single-machine scheduling problem with periodic maintenance," Computers \& Operations Research, vol. 39, no. 9, pp. 2196-2205, 2012.

[29] Y. Yin, T. C. E. Cheng, D. Xu, and C.-C. Wu, "Common due date assignment and scheduling with a rate-modifying activity to minimize the due date, earliness, tardiness, holding, and batch delivery cost," Computers and Industrial Engineering, vol. 63, no. 1, pp. 223-234, 2012.

[30] M. Dong, "Parallel machine scheduling with limited controllable machine availability," International Journal of Production Research, vol. 51, no. 8, pp. 2240-2252, 2013.

[31] C.-J. Hsu, M. Ji, J.-Y. Guo, and D.-L. Yang, "Unrelated parallelmachine scheduling problems with aging effects and deteriorating maintenance activities," Information Sciences, vol. 253, pp. 163-169, 2013.

[32] L. Shen, D. Wang, and X.-Y. Wang, "Parallel-machine scheduling with non-simultaneous machine available time," Applied Mathematical Modelling, vol. 37, no. 7, pp. 5227-5232, 2013.

[33] B. Vahedi-Nouri, P. Fattahi, M. Rohaninejad, and R. TavakkoliMoghaddam, "Minimizing the total completion time on a single machine with the learning effect and multiple availability constraints," Applied Mathematical Modelling, vol. 37, no. 5, pp. 3126-3137, 2013.

[34] D. Xu and D.-L. Yang, "Makespan minimization for two parallel machines scheduling with a periodic availability constraint: mathematical programming model, average-case analysis, and anomalies," Applied Mathematical Modelling, vol. 37, no. 14-15, pp. 7561-7567, 2013.

[35] N. Hashemian, C. Diallo, and B. Vizvári, "Makespan minimization for parallel machines scheduling with multiple availability constraints," Annals of Operations Research, vol. 213, no. 1, pp. 173-186, 2014.

[36] V. Kaplanoğlu, "Multi-agent based approach for single machine scheduling with sequence-dependent setup times and machine maintenance," Applied Soft Computing, vol. 23, pp. 165-179, 2014.

[37] K. Rustogi and V. A. Strusevich, "Combining time and position dependent effects on a single machine subject to rate-modifying activities," Omega, vol. 42, no. 1, pp. 166-178, 2014.

[38] Y. Yin, D. Ye, and G. Zhang, "Single machine batch scheduling to minimize the sum of total flow time and batch delivery cost with an unavailability interval," Information Sciences, vol. 274, pp. 310-322, 2014.

[39] Y. Yin, W.-H. Wu, T. C. E. Cheng, and C.-C. Wu, "Due-date assignment and single-machine scheduling with generalised position-dependent deteriorating jobs and deteriorating multimaintenance activities," International Journal of Production Research, vol. 52, no. 8, pp. 2311-2326, 2014.

[40] K. Rustogi and V. A. Strusevich, "Single machine scheduling with time-dependent linear deterioration and rate-modifying maintenance," Journal of the Operational Research Society, vol. 66, no. 3, pp. 500-515, 2015.

[41] C. Low, R.-K. Li, G.-H. Wu, and C.-L. Huang, "Minimizing the sum of absolute deviations under a common due date for a single-machine scheduling problem with availability constraints," Journal of Industrial and Production Engineering, vol. 32, no. 3, pp. 204-217, 2015.

[42] R. L. Graham, E. L. Lawler, J. K. Lenstra, and A. H. G. R. Kan, "Optimization and approximation in deterministic sequencing and scheduling: a survey," Annals of Discrete Mathematics, vol. 5, pp. 287-326, 1979. 
[43] T. C. E. Cheng and H. G. Kahlbacher, "A proof for the longestjob-first policy in one-machine scheduling," Naval Research Logistics, vol. 38, no. 5, pp. 715-720, 1991.

[44] I. Kacem, C. Chu, and A. Souissi, "Single-machine scheduling with an availability constraint to minimize the weighted sum of the completion times," Computers \& Operations Research, vol. 35, no. 3, pp. 827-844, 2008. 


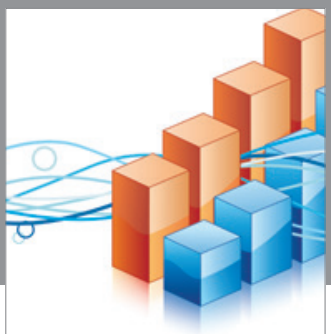

Advances in

Operations Research

vatem alat4

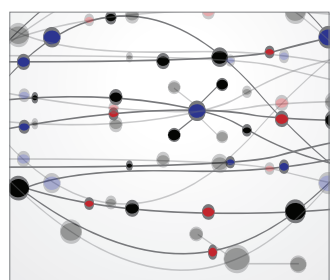

\section{The Scientific} World Journal
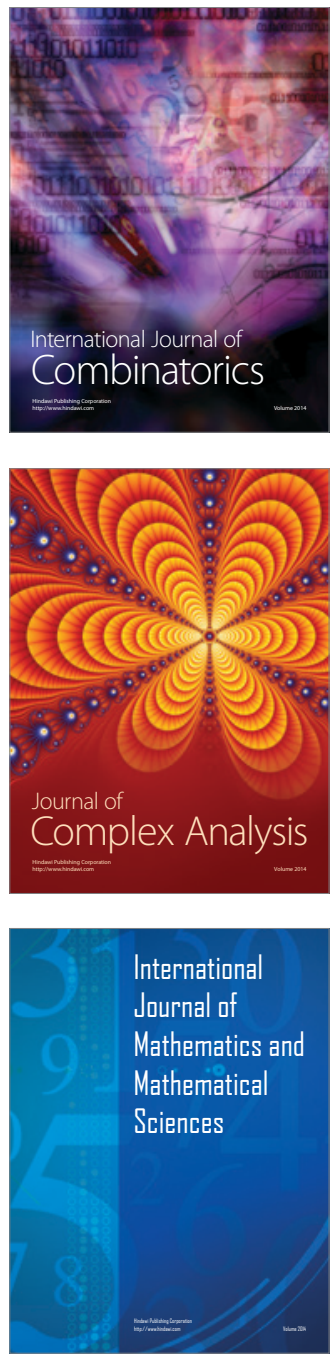
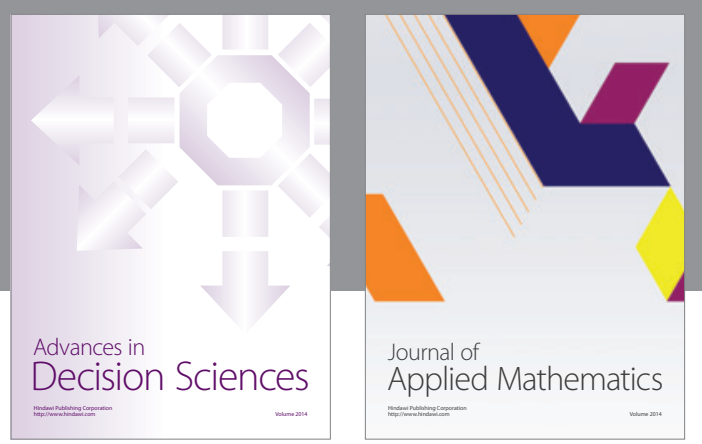

Algebra

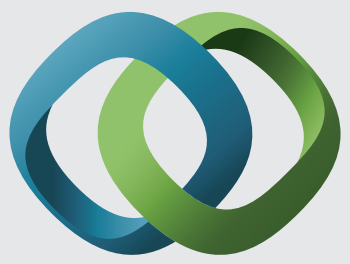

\section{Hindawi}

Submit your manuscripts at

http://www.hindawi.com
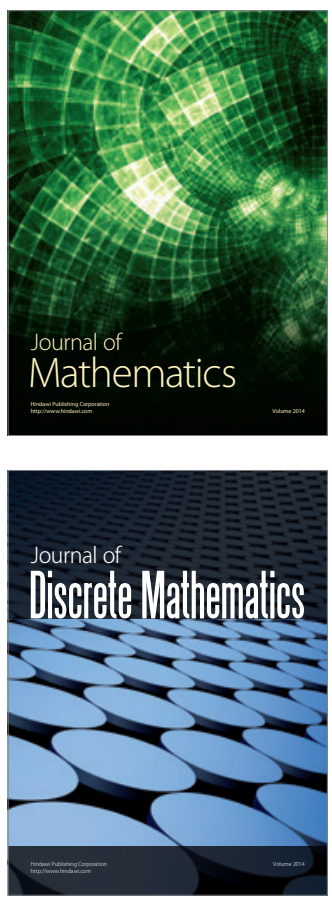

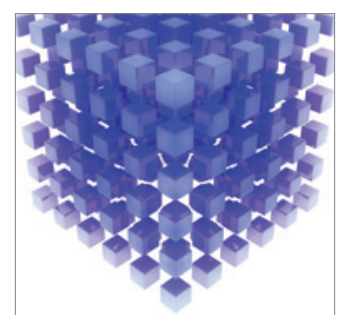

Mathematical Problems in Engineering
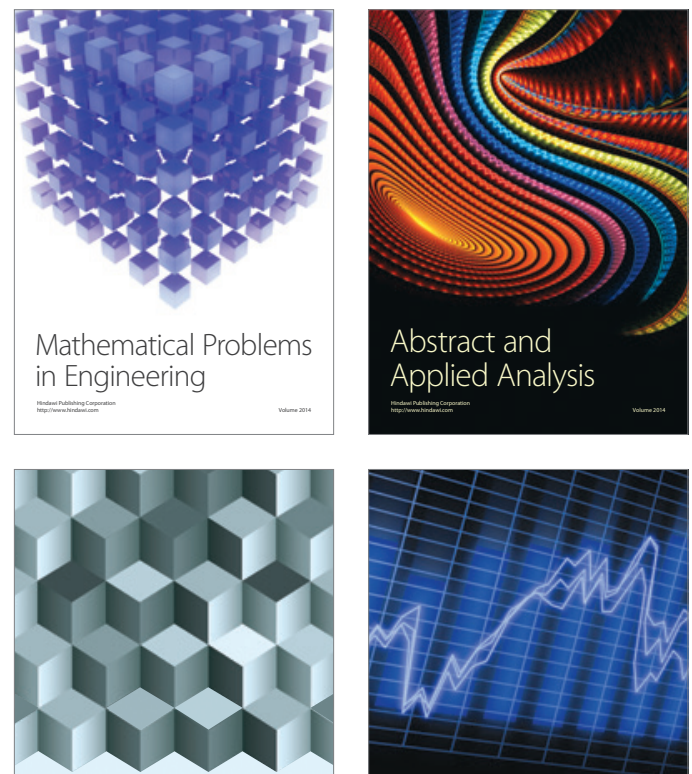

Journal of

Function Spaces

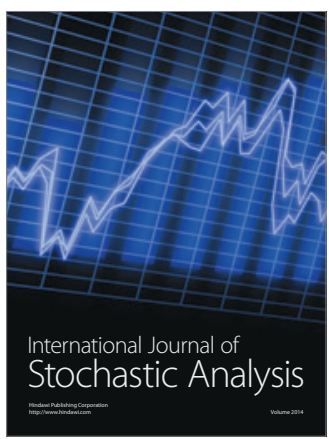

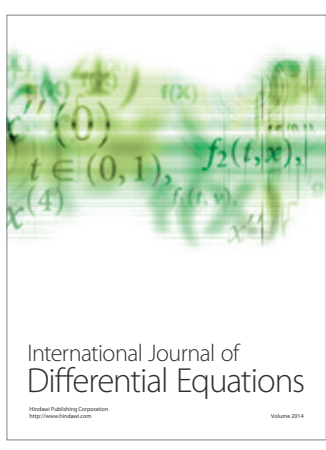
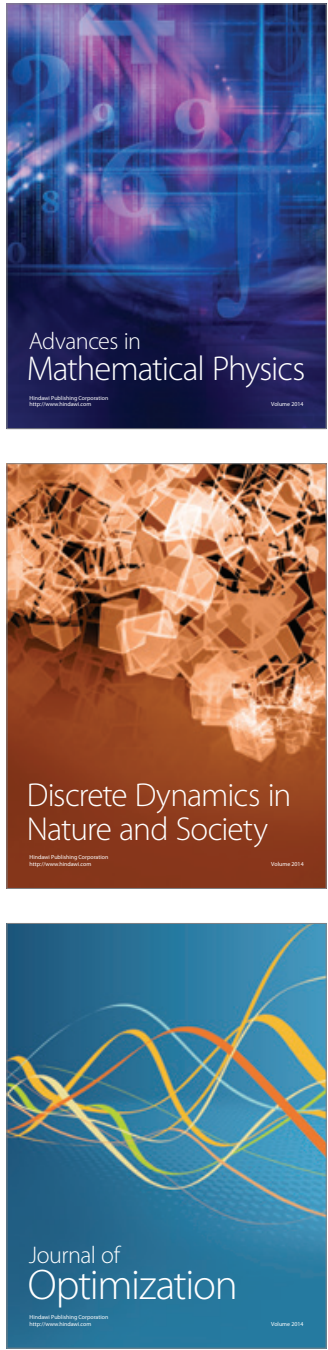